\title{
A new climate dataset for systematic assessments of climate change impacts as a function of global warming
}

\author{
J. Heinke ${ }^{1,2}$, S. Ostberg ${ }^{1}$, S. Schaphoff ${ }^{1}$, K. Frieler ${ }^{1}$, C. Müller ${ }^{1}$, D. Gerten ${ }^{1}$, M. Meinshausen ${ }^{1,3}$, and W. Lucht ${ }^{1,4}$ \\ ${ }^{1}$ Potsdam Institute for Climate Impact Research, Telegraphenberg A62, 14473 Potsdam, Germany \\ ${ }^{2}$ International Livestock Research Institute, P.O. Box 30709, Nairobi, Kenya \\ ${ }^{3}$ School of Earth Sciences, McCoy Building, University of Melbourne, Victoria 3010, Australia \\ ${ }^{4}$ Department of Geography, Humboldt-Universität zu Berlin, Berlin, Germany
}

Correspondence to: J. Heinke (jens.heinke@pik-potsdam.de)

Received: 19 October 2012 - Published in Geosci. Model Dev. Discuss.: 6 November 2012

Revised: 1 July 2013 - Accepted: 19 August 2013 - Published: 16 October 2013

\begin{abstract}
In the ongoing political debate on climate change, global mean temperature change $\left(\Delta T_{\text {glob }}\right)$ has become the yardstick by which mitigation costs, impacts from unavoided climate change, and adaptation requirements are discussed. For a scientifically informed discourse along these lines, systematic assessments of climate change impacts as a function of $\Delta T_{\text {glob }}$ are required. The current availability of climate change scenarios constrains this type of assessment to a narrow range of temperature change and/or a reduced ensemble of climate models. Here, a newly composed dataset of climate change scenarios is presented that addresses the specific requirements for global assessments of climate change impacts as a function of $\Delta T_{\text {glob. A pattern-scaling approach }}$ is applied to extract generalised patterns of spatially explicit change in temperature, precipitation and cloudiness from 19 Atmosphere-Ocean General Circulation Models (AOGCMs). The patterns are combined with scenarios of global mean temperature increase obtained from the reducedcomplexity climate model MAGICC6 to create climate scenarios covering warming levels from 1.5 to 5 degrees above pre-industrial levels around the year 2100. The patterns are shown to sufficiently maintain the original AOGCMs' climate change properties, even though they, necessarily, utilise a simplified relationships between $\Delta T_{\text {glob }}$ and changes in local climate properties. The dataset (made available online upon final publication of this paper) facilitates systematic analyses of climate change impacts as it covers a wider and finer-spaced range of climate change scenarios than the original AOGCM simulations.
\end{abstract}

\section{Introduction}

Impacts of anticipated future climate change on ecosystems and human societies are reason for major concern. Projections of such impacts are, however, characterised by uncertainties in greenhouse gas (GHG) emissions scenarios, their implementation in climate models (involving inter alia structural uncertainties of climate models) and their subsequent use in impact models. Despite intense research summarised, for example, by the Intergovernmental Panel on Climate Change's Working Group II report (Parry et al., 2007), assessments commonly lack systematic quantification of impacts as a function of global warming, as only a small and often opportunistic selection of available climate change scenarios is employed. This hampers direct comparisons between studies (e.g. Müller et al., 2011) and also our understanding of how impacts and their likelihood change over time or as a function of global mean temperature $\left(T_{\text {glob }}\right)$. The magnitude of impacts to be expected given specific degrees of $T_{\text {glob }}$ rise has gained increasing attention in recent years due to the United Nations Framework Convention on Climate Change's stipulation to prevent "dangerous climate change" and the ensuing discussion on whether this would be met by a 2 degree mitigation target (rather than, for example, a 1.5 or 3 degree target). Besides requiring an understanding of how impacts individually and collectively accumulate with increasing $T_{\text {glob }}$, an understanding of the consequences of missing a given target is important for this discussion (e.g. Mann, 2009). Compilations of individual impact studies have helped to illustrate the underlying "reasons for 
concern" (Smith et al., 2009) but do not provide the consistent quantitative information needed.

In view of the importance of mitigation targets for the debate on climate change mitigation and the substantial investments required to meet them, the number of studies that scrutinise systematically and consistently the worldwide impacts to be expected as a function of $\Delta T_{\text {glob }}$, let alone their uncertainties, is surprisingly small. Examples for global assessments of impacts ordered along $\Delta T_{\text {glob }}$ and derived with single impact modelling frameworks are those by Arnell et al. (2011); Gosling et al. (2010), and Murray et al. (2012) for freshwater availability, and those by Gerber et al. (2004); Scholze et al. (2006); Sitch et al. (2008), and Heyder et al. (2011) for ecosystems and the carbon cycle. Other assessments have focused on diverse impacts given a $\Delta T_{\text {glob }}$ of 4 degrees (see New et al., 2011).

While much of the uncertainty in $T_{\text {glob }}$ is attributable to the fact that the exact development of future GHG emissions cannot be known - requiring a scenario approach (Hawkins and Sutton, 2009) - the parameterisation of AtmosphereOcean General Circulation Models (AOGCMs) additionally contributes to uncertainty in regional temperature and precipitation changes associated with a given $\Delta T_{\text {glob }}$ (Hawkins and Sutton, 2011). Most of above-mentioned studies could account only partly for the latter, as they either relied on a small selection of AOGCMs or grouped larger ensembles according to the $\Delta T_{\text {glob }}$ reached by the individual AOGCMs by the end of their simulation period (e.g. Scholze et al., 2006). More rigorous assessments of impacts as a function of global warming are generally limited by the availability of AOGCM simulations in the CMIP3 archive. The range of warming levels covered by the different AOGCMs differs widely and the increase in $T_{\text {glob }}$ over the twenty-first century for the highest emission scenario A2 is only 3.4 in the multi-model mean (Meehl et al., 2007).

Overall, systematic assessments of climate change impacts as a function of global warming require that a large $\Delta T_{\text {glob }}$ range be covered (from, for example, 1.5 to 5 degrees), and that the respective $\Delta T_{\text {glob }}$ levels are reached at around the same time. Furthermore, for every $\Delta T_{\text {glob }}$ level, information on local changes in key climate variables (such as temperature, precipitation, radiation or cloudiness) should consider an AOGCM multi-model ensemble as large as possible, in order to account for the substantial climate model-structural uncertainty. Such consistent information is not directly available in the existing CMIP3 and CMIP5 climate databases - it requires fusion of comprehensive datasets on climate change patterns from different AOGCMs with different $\Delta T_{\text {glob }}$ trajectories (and underlying emissions trajectories), information on observed climate (without AOGCM biases), and reducedcomplexity models able to overcome the high computation requirements of AOGCMs.

To address some of these features, a number of studies (e.g. Gosling et al., 2010; Murray et al., 2012) have used emulated rather than original AOGCM output, calculated with the so-called "pattern-scaling" technique (Mitchell, 2003) that makes use of the correlation between local long-term mean changes of climate variables and $\Delta T_{\text {glob. Scaling co- }}$ efficients were found to differ spatially and seasonally, but particularly for temperature they are nearly independent of the GHG emission scenarios considered and sufficiently accurate over a wide range of $\Delta T_{\text {glob }}$ (Solomon et al., 2009; Mitchell, 2003; Huntingford and Cox, 2000). Hence, patternscaling is an efficient method to generate climate scenarios for systematic analyses of climate impacts as a function of $\Delta T_{\text {glob. }}$

Using a comprehensive pattern-scaling approach covering monthly mean surface temperature, cloudiness and precipitation, we here present a newly collated global dataset of climate change scenarios that overcomes most of the above problems and is suited for systematic, macro-scale impact assessments with empirical or process-based impact models. It is based on GCM-specific scaling patterns that are combined with time series of $\Delta T_{\text {glob }}$ generated by a reducedcomplexity climate model, MAGICC6 (Meinshausen et al., 2011a). The emissions scenarios are designed such that each of eight $\Delta T_{\text {glob }}$ levels ( 1.5 to 5 degrees above pre-industrial levels in 0.5 degree steps) is reached by 2100 . Monthly climate anomaly patterns are derived for each of 19 AOGCMs available from the World Climate Research Programme's (WCRP's) Coupled Model Intercomparison Project phase 3 (CMIP3) multi-model dataset. Scaling the derived generic change patterns per degree of global mean warming with the $\Delta T_{\text {glob }}$ trajectories generates transient time series of climate anomalies up to 2100 . This dataset enables consistent analyses of impacts as a function of $\Delta T_{\text {glob }}$ at the end of the century, and improved comparability of climate patterns and resulting impacts for given $T_{\text {glob }}$ levels. The dataset is referred to as "PanClim" (PAtterN scaling CLIMate dataset) to indicate its methodological background and its wide-spanning coverage of the scenario space (pan, Greek for "all", "involving all members"). The complete PanClim dataset is available for download from http://www.panclim.org.

\section{Methods}

Figure 1 sketches the steps of data processing and combination involved in the creation of the climate scenarios, described in detail in the following sections. Section $2.1 \mathrm{de}-$ scribes the extraction of scaling patterns - i.e. the spatial fields of local (monthly) climate change per one degree of $\Delta T_{\text {glob }}$ - from AOGCM simulations. Section 2.2 covers the generation of $T_{\text {glob }}$ trajectories by the MAGICC6 model, and their combination with the derived scaling patterns to generate time series of mean local climate anomalies for the given warming scenarios. Section 2.3 focuses on the combination of these local anomalies with data on observed variability and climatological means to generate climate scenarios 


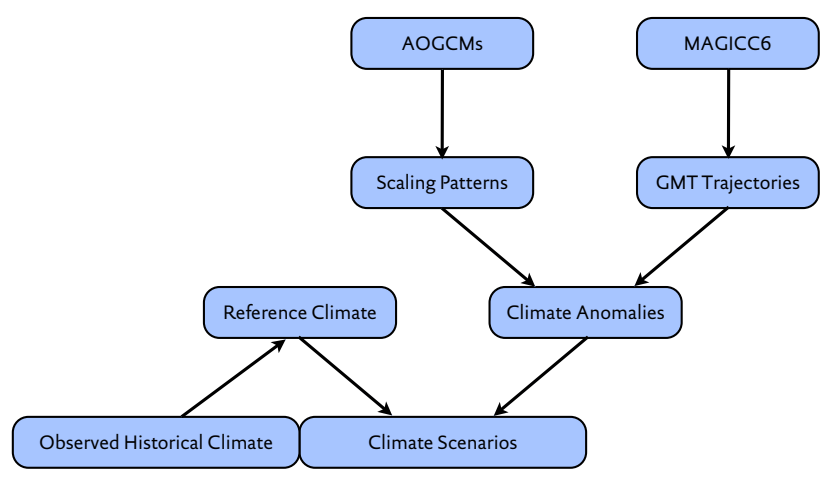

Fig. 1. Flow chart of data processing for the generation of climate scenarios.

harmonised with historical observations, covering the entire global land area.

\subsection{Derivation of scaling patterns from AOGCM simulations}

The basic concept behind the methodology described in this paper is that any simulated or observed monthly time series $V(x, m, y)$ of a climate variable $V$ (e.g. air temperature) in location $x$, month $m$, and year $y$ can be decomposed as follows:

$V(x, m, y)=\bar{V}(x, m)+\Delta \bar{V}(x, m, y)+e(x, m, y)$,

where $\bar{V}(x, m)$ denotes the long-term mean and $\Delta \bar{V}(x, m, y)$ the long-term mean change of variable $V$; the term $e(x, m, y)$ describes the natural interannual variability around the longterm mean.

The general idea of the pattern scaling approach is to relate the local anomalies in the long-term mean $\Delta \bar{V}(x, m, y)$ in Eq. (1) to a global scaler for which scenario trajectories can be easily obtained (Mitchell, 2003). In agreement with previous studies (e.g., Huntingford and Cox, 2000, Mitchell, 2003), we here use global mean temperature $\Delta T_{\text {glob }}$ as scaler and assume a linear relationship between local monthly climate anomalies $\Delta \bar{V}(x, m, y)$ and $\Delta T_{\text {glob }}(y)$ :

$\Delta \bar{V}(x, m, y)=V^{*}(x, m) \cdot \Delta T_{\mathrm{glob}}(y)$,

where $V^{*}(x, m)$ is the scaling coefficient, i.e. the change in $\bar{V}(x, m)$ per degree of $\Delta T_{\text {glob }}$ for each location and month but independent of time $(y)$. The entirety of all scaling coefficients $V^{*}(x, m)$ for a particular variable and AOGCM is referred to as scaling pattern.

Substitution of Eq. (2) in Eq. (1) and subtraction of $\bar{V}(x, m)$ from both sides of the equation gives:

$V(x, m, y)-\bar{V}(x, m)=V^{*}(x, m) \cdot \Delta T_{\mathrm{glob}}(y)+e(x, m, y)$.

Equation (3) describes all deviations of $V$ from the longterm mean $\bar{V}(x, m)$ as sum of changes in the long-term mean (expressed by the linear relationship to $\left.\Delta T_{\text {glob }}(y)\right)$ and interannual variation around the long term mean. This equation has the form of a simple linear regression model that provides the basis for estimating scaling coefficients from AOGCM simulations. For the estimation of $V^{*}(x, m)$ from AOGCM simulations, the monthly data were linearly interpolated from their original spatial resolution to the target resolution used here, a regular $0.5 \times 0.5$ arc-degree grid. Estimates of $\bar{V}(x, m)$ are obtained from the pre-industrial control run (equilibrium simulation without any anthropogenic forcing) available for all AOGCMs with lengths between 100 and 990 simulation years. Subtraction of $\bar{V}(x, m)$ from simulations with climate forcing yields deviations from the longterm climatological mean that are taken as a dependent variable in the estimation of $V^{*}(x, m)$ by linear regression. The corresponding independent variable $\Delta T_{\mathrm{glob}}(y)$ is obtained from estimates of $T_{\mathrm{glob}}(y)$ that are calculated as annual areaweighted global averages (including oceans) of $T(x, m, y)$. Since the extraction of patterns of $V^{*}(x, m)$ is based on linear regression, the residual errors $e(x, m, y)$ in Eq. (3) are in fact a mixture of interannual variability and the imperfection of the regression model. The quality of the fit obtained can thus be evaluated by comparison of residual errors and respective interannual variability estimated from the control simulation (see Sect. 3.2). We applied the above methodology to estimate scaling patterns for near-surface air temperature, cloudiness and precipitation. Additionally, we studied logarithmic precipitation to reflect an alternate assumption of exponential rather than linear precipitation change. In the logarithmic precipitation regression model, exclusion of dry months alters the estimated trend of precipitation amounts under climate change. This problem is not purely of numerical nature but highlights that the change in frequency of rain months and the change in the rainfall amounts for rain months represent qualitatively different information that should be addressed separately. Hence, we removed dry months $(<1 \mathrm{~mm}$ per month) from the linear fit (Eq. 3) of both precipitation and logarithmic precipitation so that both regression models capture the change in rainfall amounts for rain months only.

Building on the basic principle of the pattern-scaling approach, the change in frequency of rain months $(p)$ was considered separately by applying a logistic regression model, in which probabilities are logit-transformed and related to a linear predictor term, which gives a generalised linear regression model:

$$
\begin{aligned}
\operatorname{logit}(p(x, m, y)) & =\ln \left(\frac{p(x, m, y)}{1-p(x, m, y)}\right) \\
& =\beta_{0}(x, m)+\beta^{*}(x, m) \cdot \Delta T_{\operatorname{glob}}(y),
\end{aligned}
$$

where $\beta_{0}(x, m)$ and $\beta^{*}(x, m)$ denote the pre-industrial value and the scaling coefficient, respectively, for logittransformed probability of rain month occurrence in location $x$ and month $m$. For the estimation of both model coefficients from time series of dry/rain month occurrence we used the 
$\operatorname{glm}()$ function (Generalised Linear Model) from the core package "stats" of the statistical software R (R Development Core Team, 2011).

\subsection{Construction of climate scenarios from derived patterns}

\subsubsection{Construction of scenarios of global mean temperature increase}

The derived scaling patterns $V^{*}(x, m)$ for the different climate variables are the basis for constructing time series of local anomalies of climate variables consistent with prescribed $T_{\text {glob }}$ trajectories. We ran the MAGICC6 model to obtain physically and systemically plausible $\Delta T_{\text {glob }}$ trajectories and corresponding trajectories of atmospheric $\mathrm{CO}_{2}$ concentration $\left(\left[\mathrm{CO}_{2}\right]\right)$ (required for some impact models). MAGICC6 is a highly efficient reduced-complexity carbon cycle climate model (Meinshausen et al., 2011a) that has been shown to closely emulate mean results of complex AOGCMs from the CMIP3 data base (Meinshausen et al., 2011b). Here, MAGICC6 was used to calculate $\Delta T_{\text {glob }}$ and $\left[\mathrm{CO}_{2}\right]$ for a large number of artificial emissions pathways, constructed as described by (Meinshausen et al., 2009). For that purpose MAGICC's carbon cycle parameters were adjusted to reproduce the Bern carbon cycle model and the climate model parameters were chosen to reproduce the median responses of the CMIP3 AOGCM ensemble. Climate sensitivity, for example, was set to $3.0 \mathrm{~K}$.

From the generated large ensemble of pathways we selected those pairs of $\Delta T_{\text {glob }}$ and $\left[\mathrm{CO}_{2}\right]$ trajectories where average $\Delta T_{\text {glob }}$ in the period 2086-2115 reached 1.5, 2.0, 2.5, $3.0,3.5,4.0,4.5$, and 5.0 degrees above the pre-industrial level (see Fig. 2). The definition of the temperature target for a period rather than for a single year (e.g. 2100) was chosen because the analysis of time periods is common practice in impact assessments to avoid spurious effects from interannual variability. $30 \mathrm{yr}$ is a typical length used in impact studies in hydrology, agriculture, and ecosystems, for which our new data set is designed.

An outstanding feature in Fig. 2 that illustrates the abovementioned physical and systemic plausibility is the initially stronger increase in $T_{\text {glob }}$ in the lower than in the high temperature scenarios. Stronger mitigation scenarios tend to show a much faster decrease in aerosol emissions than in $\mathrm{CO}_{2}$ emissions, as a rapid decrease of $\mathrm{CO}_{2}$ emissions is accompanied by a switch to "cleaner" sources of energy. This correlation between $\mathrm{CO}_{2}$ and aerosol emissions results from our use of the Equal Quantile Walk method (Meinshausen et al., 2006) to create the different emission profiles that led to the various warming levels. The drop in aerosol emissions in combination with the much shorter residence time of aerosols in the atmosphere results in a rapid reduction of the aerosol cooling effect (see Ramanathan and Feng, 2008). As a consequence, the committed warming from current $\left[\mathrm{CO}_{2}\right]$
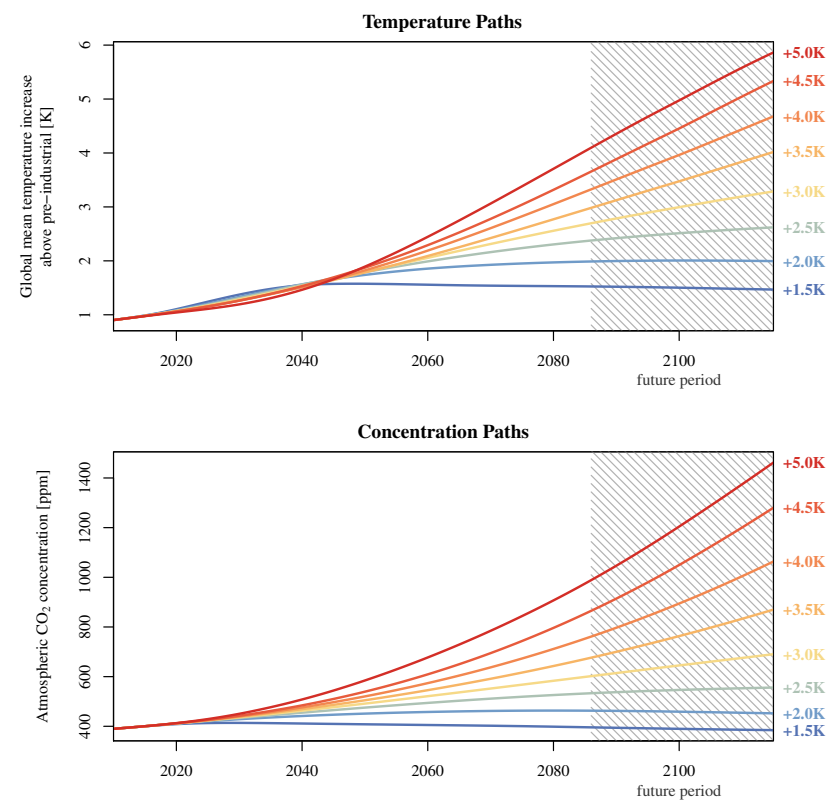

Fig. 2. Trajectories of global mean temperature increase used in this study and corresponding atmospheric $\mathrm{CO}_{2}$ concentrations from the MAGICC6 model. The shaded area indicates the the time period for which the temperature targets are calculated.

can unfold before a further reduction of $\mathrm{CO}_{2}$ emissions eventually results in an overall decrease in radiative forcing and temperature. Conversely, the $\mathrm{CO}_{2}$ emissions in the high temperature scenarios are accompanied by high aerosol emissions that maintain the cooling effect. Besides the possibility to produce $T_{\text {glob }}$ scenarios together with consistent $\left[\mathrm{CO}_{2}\right]$ trajectories, the consideration of such effects is the major advantage of applying MAGICC6 in this study.

\subsubsection{Construction of local time series of climate anomalies}

Local time series of climate anomalies $\Delta \bar{V}_{\text {scen }}(x, m, y)$ for the four climate variables were obtained by multiplying the scaling coefficients $V^{*}(x, m)$ with the $\Delta T_{\text {glob }}(y)$ trajectories for each scenario (Eq. 2). Because the obtained time series of anomalies are combined with climate observations in the next step (see Sect. 2.3), it is necessary to account for the climate change signal already present in these observations. Anomalies are therefore calculated relative to the last year of observations, 2009. This is achieved by subtracting the $T_{\text {glob }}$ increase above pre-industrial level for the year 2009 $(\sim 0.9 \mathrm{~K})$ from the $T_{\text {glob }}$ trajectories of the MAGICC6 scenarios before multiplying them with the anomaly patterns. In all cases, anomalies were only calculated if the significance level of the slope of the regression model is $>0.9$; otherwise they were set to zero.

For temperature, the obtained local anomalies can be used without any restriction. In the case of cloudiness and 
precipitation, however, the obtained anomalies may result in an exceedence of the lower and, in the case of cloudiness, also the upper limit of possible values for these variables. For cloudiness this problem is less critical as it is not used directly in impact models but serves, among other parameters, as a proxy for atmospheric transmissivity and emissivity in the estimation of radiation budgets. We therefore consider a simple capping of anomalies to prevent the exceedance of upper and lower limits, a sufficiently accurate solution. In contrast to cloudiness, precipitation is an essential variable and calculation of anomalies that would result in physically implausible negative precipitation rates should be avoided from the beginning. Anomalies for decreasing precipitation are therefore estimated from the regression models for logarithmic precipitation, which is equivalent to the assumption of exponential precipitation decrease. As there is no indication that precipitation would increase exponentially with $T_{\text {glob }}$, precipitation increases are estimated from the linear regression models for untransformed precipitation. For small change rates, the linear and the exponential approach yield very similar anomalies, while for large change rates the linear approach avoids unrealistically augmented increases and the exponential approach avoids negative precipitation rates (see also Watterson, 2008). For estimating rain month frequency anomalies, changes in the linear predictor term of Eq. (4), i.e. anomalies of logit probabilities, were calculated. These obtained anomalies can be used without restrictions, as the range of logit probabilities is unconstrained. For the transformation into actual frequency anomalies see Sect. 2.3.4.

\subsection{Creation of climate scenarios from observed climate and derived climate anomalies}

In order to obtain complete scenario time series of climate variables $V_{\text {scen }}(x, m, y)$ that can be used for transient impact model simulations, the local scenario time series of climate anomalies $\Delta \bar{V}_{\text {scen }}(x, m, y)$ are combined with time series here referred to as "reference time series" $V_{\text {ref }}(x, m, y)$ - that provide the long-term climatological mean $\bar{V}(x, m)$ and interannual variability $e(x, m, y)$ (Eq. 1$)$. Reference time series for temperature and cloudiness are constructed from and are consistent with the CRU TS3.1 global climate dataset (Harris et al., 2013); reference time series for precipitation are based the GPCC full reanalysis dataset version 5 (Rudolf et al., 2010).

Because GPCC and CRU datasets have a slightly different land mask, the GPCC dataset was adjusted to the CRU land mask (67 420 grid cells) by filling up missing cells by interpolation. For this, the five neighbouring cells with the highest weight - calculated from distance and angular separation (New et al., 2000) - within a $450 \mathrm{~km}$ radius were used. If $<5$ values were available, the interpolation was performed on this reduced data basis; if $<2$, the precipitation from the CRU TS3.1 dataset was used. Grid cells only present in the GPCC land mask but not in the CRU land mask were excluded. Altogether, 767 grid cells were introduced by interpolation, 298 grid cells were directly taken from CRU TS3.1, and 1013 grid cells were omitted from the GPCC dataset.

106-yr reference time series covering the scenario period (2010-2115) were composed as a random sequence of years from historical observations of the period 1961-2009. To preserve interannual autocorrelation, spatial coherence, and correlation among climate variables, all months and grid cells for all climate variables were taken from the same year. Prior to resampling, the trend in temperature was removed in a way that the detrended time series of temperature are representative for the climatologic mean of year 2009 obtained from the trend analysis. In the process of data preparation, observations of precipitation and cloudiness were found to exhibit strong interannual/interdecadal variability, which negatively affects the robustness of estimated trends. In order to avoid spurious effects from removing these trends, the original data were used directly for generating the reference time series for cloudiness and precipitation. The time series of resampled observations obtained are assumed to represent variability and climatology for the reference year 2009, to be consistent with the reference year for the derived anomalies. This consistency between the constructed reference time series, derived anomaly time series, and observations allows for seamless combination of historic observations with future climate projections and thus for transient impact model runs.

The combination of the anomalies with the reference time series is a crucial step and related to the general problem of whether to apply climate anomalies as an absolute change:

$V_{\text {scen }}(x, m, y)=V_{\text {ref }}(x, m, y)+\Delta \bar{V}_{\text {scen }}(x, m, y)$

or a relative change:

$V_{\text {scen }}(x, m, y)=V_{\text {ref }}(x, m, y) \cdot \frac{\bar{V}_{\text {base }}(x, m)+\Delta \bar{V}_{\text {scen }}(x, m, y)}{\bar{V}_{\text {base }}(x, m)},(6)$

where $\bar{V}_{\text {base }}(x, m)$ is the basis for the anomalies in the AOGCM, i.e. the long-term climatological mean of the AOGCM's representation of present-day climate. Where biases in the AOGCM's representation of present-day climate are small, the application of anomalies as relative change imposes a similar mean change to the scenario time series than the application as absolute change. That is, the difference between the mean of the scenario time series and the reference time series is similar to the original anomaly. As biases increase, climate anomalies are progressively altered with the relative approach. This alteration is an expression of the adjustment of the absolute anomaly derived from a biased base level in the AOGCM to the observed level, which is the actual motivation for using the relative approach. The relevance of this adjustment is particularly apparent where decreases from overestimated levels in the AOGCM are applied to lower observed levels. Without the attenuation of the anomaly by the relative approach the application of a negative change might well lead to negative values. However, for the reverse case - 
increases from underestimated levels - this approach is less favourable as it may lead to an unrealistic augmentation of the absolute anomaly.

Another difference between the two approaches is that with the absolute application of anomalies interannual variability remains unchanged, while with the relative application interannual variability is altered in a way that the coefficient of variation remains constant. The relevance of this variability adjustment is most apparent for cases where negative anomalies bring the mean of the scenario time series close to zero. In these cases a corresponding decrease of variability is required to prevent the occurrence of negative values.

The procedures used to apply the anomaly time series to the reference time series for different climate variables are described in the remaining part of this section. In order to improve readability, the parameters $x$ and $m$ are omitted; only the parameter $y$ is used to differentiate terms that vary over time from time-invariant terms. Thus, Eqs. (7)-(14) can be seen to describe the processes for a particular location $x$ and month $m$ but apply to all locations and months.

\subsubsection{Temperature}

Since temperature biases in AOGCMs are very small compared to absolute temperature levels, the application as absolute or relative change would give very similar results. However, temperature anomalies are commonly treated as absolute changes in the literature and are thus applied as absolute change here:

$T_{\text {scen }}(y)=T_{\text {ref }}(y)+\Delta \bar{T}_{\text {scen }}(y)$,

where $T_{\text {scen }}(y), T_{\text {ref }}(y)$, and $\Delta \bar{T}_{\text {scen }}(y)$ are the temperature time series of the scenario, the reference time series, and the time series of anomalies, respectively.

\subsubsection{Cloudiness}

For cloudiness, anomalies were applied as relative changes. Due to the problem of augmentation of anomalies when applied as relative change to higher observed levels, there is a risk of exceeding the upper $100 \%$ limit in these cases. Increases in cloudiness are therefore applied as relative decreases of cloudlessness, i.e. $100 \%$ - cloudiness:

$$
\begin{aligned}
& \operatorname{Cld}_{\text {scen }}(y)= \\
& \left\{\begin{array}{ll}
\operatorname{Cld}_{\text {ref }}(y) \cdot \frac{\overline{\operatorname{Cld}}_{\text {base }}+\Delta \overline{\operatorname{Cld}}_{\text {scen }}(y)}{\overline{\operatorname{Cld}}_{\text {base }}} & \text { for } \Delta \overline{\operatorname{Cld}}_{\text {scen }}(\mathrm{y})<0 \\
100-\left(100-\operatorname{Cld}_{\text {ref }}(y)\right) \cdot \frac{100-\left(\overline{\operatorname{Cld}}_{\text {base }}+\Delta \overline{\operatorname{Cld}}_{\text {scen }}(y)\right)}{100-\overline{\operatorname{Cld}}_{\text {base }}} & \text { for } \Delta \overline{\operatorname{Cld}}_{\text {scen }}(\mathrm{y})>0
\end{array},(8)\right.
\end{aligned}
$$

with $\operatorname{Cld}_{\text {scen }}(y), \operatorname{Cld}_{\text {ref }}(y), \Delta \overline{\operatorname{Cld}}_{\text {scen }}(y)$, and $\overline{\operatorname{Cld}}_{\text {base }}$ denoting the cloudiness time series of the scenario, the reference time series, the time series of anomalies, and the present-day climatological mean cloudiness in the AOGCM, respectively. For consistency with the anomalies and the reference time series, $\overline{\mathrm{Cld}}_{\text {base }}$ needs to represent the simulated climatological mean for the year 2009. It is estimated by adding the cloudiness anomaly for a $0.9 \mathrm{~K}$ warming to the climatological mean of the control run (see Sect. 2.2.2).

\subsubsection{Precipitation}

The application of precipitation anomalies is particularly challenging because of the importance of precipitation as key variable in impact assessments and the partially very large biases in simulated present-day precipitation. In cases where simulated precipitation in the control run is very low, small absolute increases correspond to very large relative changes. When applied to significantly higher observed precipitation rates, the absolute changes can become unrealistically large. Other studies have therefore proposed to use absolute changes or limit the relative changes in such cases (Carter et al., 1994; Hulme et al., 1995). Füssel (2003) notes that the problem depends on the degree of underestimation of present-day precipitation rates by AOGCMs and proposes a seamless transition from a relative towards an absolute application of anomalies, depending on the degree of underestimation. Here we adopt the approach by Füssel (2003) with some modifications required for the application to time series (see also Gerten et al., 2011, where a similar approach was used). Anomalies are applied as relative change, but as the underestimation of present-day precipitation in the AOGCM increases, the applied relative change is reduced so that the resulting mean change in the scenario time series becomes increasingly similar to the absolute change:

$P_{\text {scen }}(y)=P_{\text {ref }}(y) \cdot\left[1+\left(\frac{\Delta \bar{P}_{\text {scen }}(y)}{\bar{P}_{\text {ref }}}\right)\left(\frac{\bar{P}_{\text {ref }}}{\bar{P}_{\text {base }}}\right)^{\lambda}\right]$,

with

$\lambda=\left\{\begin{array}{ll}\sqrt{\frac{\bar{P}_{\text {base }}}{\bar{P}_{\text {ref }}}} & \text { for } \overline{\mathrm{P}}_{\text {base }}<\overline{\mathrm{P}}_{\text {ref }}, \\ 1 & \text { for } \overline{\mathrm{P}}_{\text {base }} \geq \overline{\mathrm{P}}_{\text {ref }}\end{array}\right.$,

with $P_{\text {scen }}(y), P_{\text {ref }}(y)$, and $\Delta \bar{P}_{\text {scen }}(y)$ denoting the precipitation time series of the scenario, the reference time series, and the time series of anomalies, respectively; and $\bar{P}_{\text {ref }}$ and $\bar{P}_{\text {base }}$ denoting the climatological means of the reference time series and the year 2009 in the AOGCM, respectively. Estimation of $\bar{P}_{\text {base }}$ is analogous to estimation of $\overline{\mathrm{Cld}}_{\text {base }}$ (see Sect. 2.3.2). The exponent $\lambda$ determines the degree to which an anomaly is applied as absolute or relative change. If $\lambda=1$, Eq. (9) is equivalent to the relative interpretation of precipitation anomalies. If present precipitation is underestimated by the AOGCM, lower values of $\lambda$ diminish the applied relative anomaly. If $\lambda$ approaches zero, the factor applied to the values of the reference time series results in a shift of its mean equal to the absolute anomaly $\Delta \bar{P}_{\text {scen }}(y)$. Because all anomalies are applied as a factor, the coefficient of variation is preserved in the scenario time series, which implies changes in interannual variability. 


\subsubsection{Rain month frequency}

Based on the logistic regression model estimated from the AOGCM simulations, the probability of rain month occurrence was estimated for each month of the scaled scenario time series as follows:

$p_{\text {scen }}(y)=\frac{e^{z}}{1+e^{z}} \quad$ with $\quad z=\operatorname{logit}\left(p_{\text {ref }}\right)+\beta^{*} \cdot \Delta T_{\text {glob }}(y)$,

where $p_{\text {scen }}(y)$ is the probability of year $y$ in the scenario to be a rain month and $p_{\text {ref }}$ the probability of rain month occurrence in the reference time series - i.e. the fraction of rain months in that series. In cases where $p_{\text {ref }}$ is either 0 or $1, \operatorname{logit}\left(p_{\text {ref }}\right)$ cannot be calculated and was set to a value of -7 and 7 , respectively. This is equivalent to values for $p_{\text {ref }}$ of about $1 / 1100$ and $1-1 / 1100$, respectively. The term $\beta^{*} \cdot \Delta T_{\text {glob }}(y)$ denotes the anomaly of the logit rain month probability estimated from the logistic regression model and $T_{\text {glob }}$ anomalies (see Sect. 2.2.2). Because the intercept and the slope of the logistic regression model are both estimated by fitting the model to the scenario data, extreme values are sometimes obtained for $\beta^{*}$ where rain month probability is 0 or 1 and some singular dry or rain months occur towards the higher end of the temperature range. When used with the estimated intercept $\beta_{0}$, these slopes correspond to very small changes in rain month probability but produce unrealistically augmented probability changes when applied to $p_{\text {ref }}$ in Eq. (11). In order to avoid this effect, only slopes with a corresponding estimate for the intercept between -7 and 7 were applied; otherwise no change was applied. This rule applied to about $5.5 \%$ of all significant estimates for $\beta^{*}$.

The application of $p_{\text {scen }}$ to the reference time series entails the removal of excess and the introduction of additional rain months by means of a stochastic process. For this procedure, a random sequence $w(y)$ of uniformly distributed numbers between 0 and 1 is generated, which serves as a decision criterion on whether a rain month is introduced or removed in year $y$. If $p_{\text {scen }}(y)$ is smaller than $p_{\text {ref }}$ a rain month is removed if

$w(y) \geq \frac{p_{\text {scen }}(y)}{p_{\text {ref }}}$.

Conversely, if $p_{\text {scen }}(y)$ is larger than $p_{\text {ref }}$, a rain month is introduced if

$1-w(y) \geq \frac{1-p_{\text {scen }}(y)}{1-p_{\text {ref }}}$.

The precipitation event to be introduced is randomly chosen from the precipitation distribution of the respective reference time series. In cases where the reference time series has no rain month at all, a synthetic rainfall distribution is generated by interpolation from up to five neighbour cells with at least one precipitation event in their distribution. The selection criterion for these cells was taken to be the highest interpolation weight from all cells within a radius of $450 \mathrm{~km}$.
Interpolation weights were calculated as in New et al. (2000) with account for distance and angular separation.

In order to preserve the spatial and temporal coherence of the precipitation field, the same random number sequence $w(y)$ was used for all grid cells and months of the year. The rationale behind this procedure is that for neighbouring cells with similar $p_{\text {scen }}(y)$ and $p_{\text {ref }}$, rain months get removed or inserted in the same year. In order to avoid an overlap with the removal of rain months, however, the reflected sequence $1-w(y)$ was used as decision criterion for the introduction of rain months. The procedure was applied prior to the scaling of precipitation amounts described in the preceding sections. Average reference precipitation used in Eqs. (9) and (10) was calculated from this modified reference time series.

\subsubsection{Wet-day frequency}

An additional information required by many impact models is the number of wet days per month. Due to the sparse availability of daily rainfall data from AOGCMs and strong biases in frequency distribution of rainfall intensities in many AOGCMs, this information is hard to extract from these models. The number of wet days per month is therefore estimated based on New et al. (2000) using the relationship between monthly precipitation sum and number of wet days:

$\mathrm{WD}(y)=\overline{\mathrm{WD}}_{\mathrm{obs}}\left(\frac{P(y)}{\bar{P}_{\mathrm{obs}}}\right)^{\gamma}$,

where $P(y)$ and $\mathrm{WD}(y)$ represent the time series for precipitation sum and the estimated number of wet days of a month and grid cell, respectively. The exponent $\gamma$ is assumed to be 0.45 , which was found by New et al. (2000) to yield best results. The values $\overline{\mathrm{WD}}_{\text {obs }}$ and $\bar{P}_{\text {obs }}$ represent the observed 1961-1990 mean monthly wet-day frequency and precipitation sum, respectively. The former was derived from CRU TS3.1 (Harris et al., 2013) and the latter from GPCC version 5 (Rudolf et al., 2010). The means were calculated over the entire 30-yr period, including totally dry months. Because the datasets for wet days and precipitation are based on different station networks they are not fully consistent, i.e. there are cases where rain months have zero wet days (and vice versa). The absolute minimum for $\overline{\mathrm{WD}}_{\mathrm{obs}}$ is the fraction of rain months in the 30-yr period, which means that at least one wet day has to exist for each rain month. If the estimate of $\overline{\mathrm{WD}}_{\mathrm{obs}}$ is smaller than that, it was set to that minimum. This estimation procedure delivers conservative estimates of wet-day frequency for the scenario period, since the relationship between wet-day frequency and monthly precipitation sum is assumed to be constant over time. 


\section{Results and discussion}

\subsection{Properties of scaling patterns extracted from AOGCM simulations}

The scaling patterns extracted from AOGCM simulations are the core component of the scenario-building described in this paper. They provide information on spatial and temporal heterogeneity of climate change signals for primary climate variables as projected by different AOGCMs. In this section, an overview is given of the spatial coverage of fits that are significant and of basic properties of the derived patterns (mean and standard deviation). The focus is primarily on a comparison of the different climate variables with some indication of the inter-model spread. A comprehensive overview with values for individual AOGCMs is presented in Table 1.

An apparent difference between the climate variables is the spatial and temporal coverage of significant slope parameters of the regression models obtained from the AOGCM simulation. As described in Sect. 2.2.2, only slope estimates with a statistical significance $>0.9$ were accepted and used for the scaling. Each slope estimate is representative for a specific area (size of grid cell) and a specific time period of the year (length of month). In order to assess the spatial and temporal coverage of significant slope estimates, the product of area and duration for each significant slope is calculated and summed up. The sum is related to the product of total land area and length of the year to arrive at a percentage of spatial and temporal coverage.

Averaged over all AOGCMs, spatial and temporal coverage of significant slopes is $99.9 \%, 82.0 \%$, and $78.2 \%$ for temperature, cloudiness and precipitation, respectively (value for precipitation composed of $46.9 \%$ significant increases in the linear case and $31.3 \%$ significant decreases in the logarithmic case; Table 1). The average coverage of significant slopes for the logistic regression models for rain month probability is $10.9 \%$ and $10.3 \%$ if regression models with extreme intercepts are excluded (see Sect. 2.3.4). Although there is considerable variation in spatial coverage of significant fits among individual AOGCMs (see Table 1), the relative magnitude of coverage for the different variables is consistent over all models. Near full coverage is found for temperature, followed by moderate to high coverage for cloudiness and precipitation (including both increases and decreases). Coverage of significant precipitation increases is in all cases higher than for decreases although values are similar in some cases. In all cases, coverage of significant changes of rain month frequency is smallest.

Although the coverage of significant changes for cloudiness, precipitation, and rain month frequency is significantly lower than for temperature, this must not be interpreted as an indication of limited applicability of the pattern-scaling approach for these variables. A major difference between temperature and the other variables is that for the former only positive trends occur, while the other variables display a mixture of positive and negative trends (see Figs. 3-6). This implies the existence of transition zones between areas with positive and negative trends in the monthly fields where trends are de facto zero and therefore no significant slopes can be found. In addition, cloudiness and precipitation both exhibit strong interannual variability that tends to mask weak trends that primarily occur around such transition zones. Similarly, the estimation of parameters of the logistic regression model for change of rain month frequency is hampered by the stochastic nature of this variable. Moreover, vast areas with a rain month frequency of $100 \%$ (e.g. in the high latitudes and the wet tropics) remain unaffected by the occurrence of dry months under climate change (Fig. 6).

For each derived anomaly pattern two statistics - mean and standard deviation - are calculated in order to characterise the patterns. We took into account the spatial and temporal coverage of the individual slopes - i.e. by weighting them with the respective cell area and length of month. Because the aim is to illustrate the properties of the entire pattern as it is applied, grid cells and months without a significant slope are included as zero values.

Averaged over all AOGCMs the mean anomaly of temperature increase over land is estimated to be $1.32 \mathrm{~K}$ per $1 \mathrm{~K}$ increase of $T_{\text {glob }}$ (from $14.0^{\circ} \mathrm{C}$ in the reference time series). Because $T_{\text {glob }}$ anomalies and local temperature anomalies used in the regression are estimated from the same data, the value demonstrates that the land surface heats up much more than the whole of the global surface. This phenomenon is well known and is caused by the higher heat storage capacity of the oceans, which cause them to heat up less (Lambert and Chiang, 2007). Although temperature trends are found to be always positive over land (Fig. 3), there is considerable heterogeneity in the degree of warming in different regions and times of the year. This heterogeneity is captured by the pattern's standard deviation, which on average over all AOGCMs is $0.5 \mathrm{~K}$. The mean and standard deviation for individual models are in the range of 1.18-1.43 and 0.400.63 , respectively (Table 1 ).

The prevalence of a clear mean signal in the pattern is unique to temperature among the variables considered here. For cloudiness the average pattern mean is $-0.49 \%$ - less than $1 \%$ of the mean cloudiness over land in the reference time series $(55.3 \%)$. The relatively small mean change is contrasted by a higher standard deviation of $1.55 \%$, which reveals the distinct spatial and temporal pattern of changes in cloudiness. This is consistent over all individual AOGCMs, which are characterised by mean changes between -1.19 and $0.37 \%$, and pattern standard deviations between 0.97 and $2.09 \%$, respectively.

For the calculation of pattern mean and standard deviation for precipitation, the decreases of logarithmic precipitation that make up the decreasing part of the pattern need to be converted to absolute changes in precipitation. Although the nonlinearity of exponential decrease may lead to an 


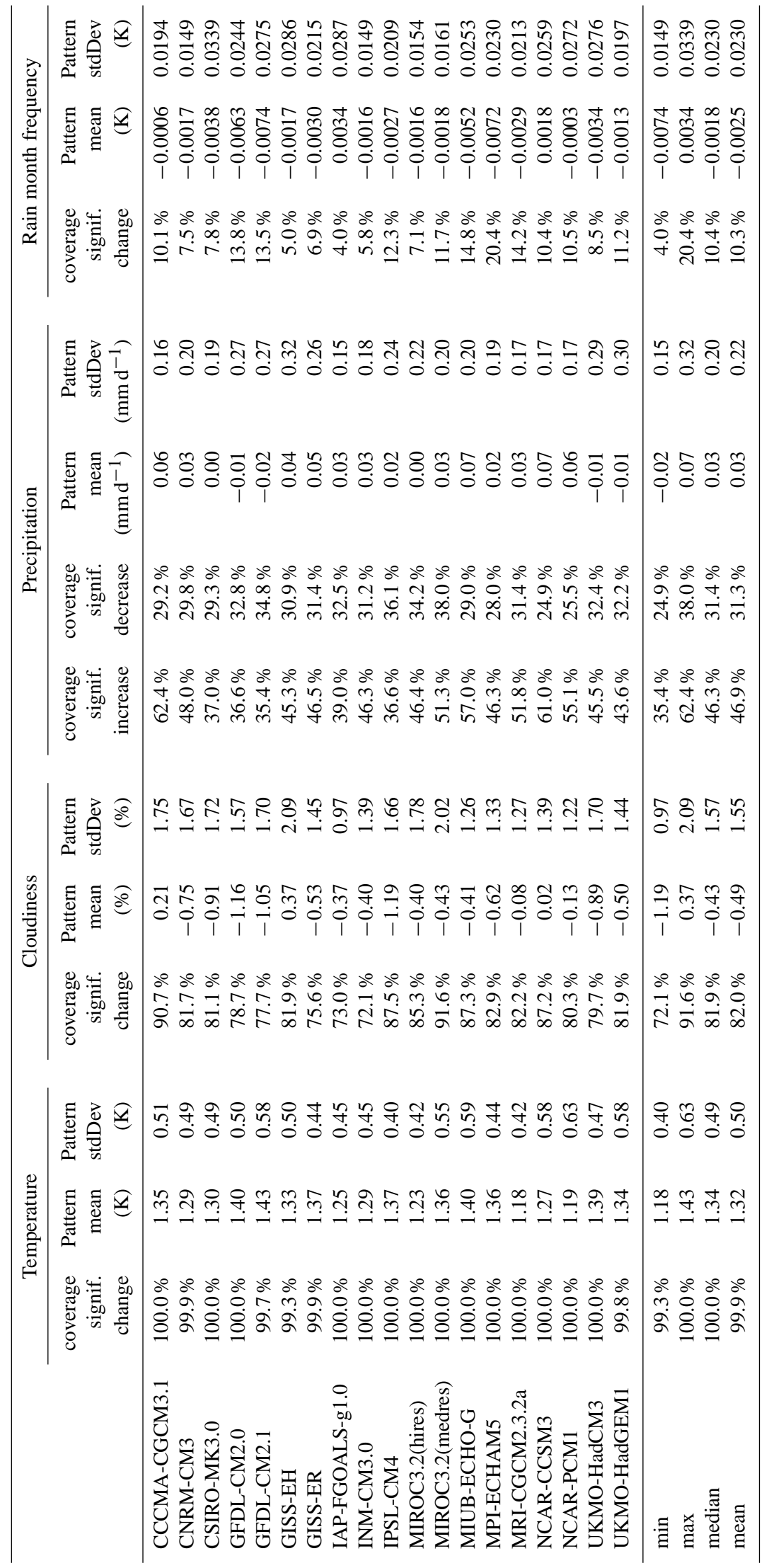




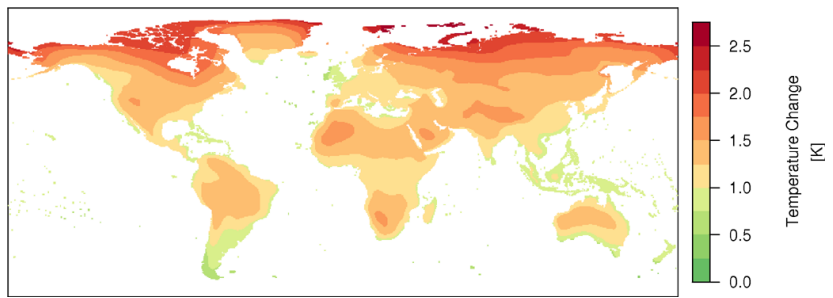

Fig. 3. Multi-model mean of the actual applied annual mean change in near surface air temperature in $\mathrm{K}$ per $1 \mathrm{~K}$ of $\Delta T_{\text {glob }}$ (Eq. 16).

augmentation of precipitation decreases, the effect remains small due to the small magnitude of slopes of logarithmic precipitation decrease $(-0.10$, average over all AOGCMs). Averaged over all AOGCMs a mean precipitation change of $0.026 \mathrm{~mm} \mathrm{~d}^{-1}$ (millimetre per day) is found, which is equivalent to $\sim 1 \%$ of the mean precipitation rate over land in the reference time series $\left(2.27 \mathrm{~mm} \mathrm{~d}^{-1}\right)$. Similar than for cloudiness, this small mean change is contrasted by a much larger standard deviation of $0.22 \mathrm{~mm} \mathrm{~d}^{-1}$ (averaged over all AOGCMs). Corresponding values for individual AOGCMs range between -0.016 and $0.069 \mathrm{~mm} \mathrm{~d}^{-1}$, and between 0.15 and $0.32 \mathrm{~mm} \mathrm{~d}^{-1}$ for mean and standard deviation, respectively (Table 1).

The slopes of the logistic regression for changes in rain month frequency are difficult to interpret in their original form and were therefore converted to changes in the fraction of rain months for the calculation of statistics. Averaged over all AOGCMs the mean change is -0.0025 rain months per month, which corresponds to an average loss of one rain month in about $33 \mathrm{yr}$ on the entire land surface (including areas with no change). Average standard deviation of rain month changes is 0.028 rain months per month. For individual AOGCMs mean rain month frequency changes are between -0.0074 and 0.0034 rain months per month with standard deviations between 0.015 and 0.034 .

\subsection{Significance of scaling patterns extracted from AOGCM simulations}

The assumption of a linear relationship between change in $T_{\text {glob }}$ and mean local change of a climate variable $V$ considered is central to pattern scaling. Although it is generally accepted that this assumption holds well for temperature (Mitchell, 2003), it may not be fully valid for other climate variables. The focus of this section is therefore on a comparison between the different variables rather than between the different AOGCMs. However, values for individual AOGCMs are presented in Table 2.

For ordinary linear square models, such as those fitted to the AOGCM data for pattern extraction, the total sum of squares (TSS) equals the sum of explained sum of squares (ESS) and residual sum of squares (RSS). For the pattern extraction, this is described in Eq. (15).

$$
\begin{aligned}
\sum_{y=1}^{N}[\Delta V(x, m, y)]^{2} & =\sum_{y=1}^{N}\left[V^{*}(x, m) \cdot \Delta T_{\mathrm{glob}}(y)\right]^{2} \\
& +\sum_{y=1}^{N}\left[\Delta V(x, m, y)-V^{*}(x, m) \cdot \Delta T_{\mathrm{glob}}(y)\right]^{2}
\end{aligned}
$$

Based on this relationship, it is possible to evaluate the significance of the extracted patterns by comparing the explained sum of squares $\sum_{y=1}^{N}\left[V^{*}(x, m) \cdot \Delta T_{\mathrm{glob}}(y)\right]^{2}$ to the total sum of squares $\sum_{y=1}^{N}[\Delta V(x, m, y)]^{2}$ to provide a measure of explained variance. However, this measure is incomplete without an analysis of how much of the residual sum of squares $\sum_{y=1}^{N}\left[\Delta V(x, m, y)-V^{*}(x, m) \cdot \Delta T_{\operatorname{glob}}(y)\right]^{2}$ can be attributed to interannual variability inherent to the climate system. This variability cannot be captured by the linear regression, and the separation of the climate signal from the background variability is in fact the basic principle of the pattern-scaling approach. For the analysis of the residual sum of squares the variance of the control run $\operatorname{Var}_{\text {cntrl }}(x, m)$ was multiplied with the number of values $N$ in the residual sum of squares to obtain an estimate of the total sum of squared interannual variability to be expected in the scenario data.

Because Eq. (15) is valid for every single regression model, the evaluation metrics derived from its terms can be calculated for every model, grid cell, and month. In order to facilitate a comparison of the performance for different variables, area-weighted means over all land cells for the different square sums are calculated for each model and month and then again averaged.

For the ratio of explained sum of squares to total sum of squares (ESS/TSS), ensemble means of 0.78, 0.20, 0.16, and 0.15 are found for temperature, cloudiness, precipitation (increases only), and logarithmic precipitation (decreases only), respectively. Corresponding ensemble means for ratios of residual mean of squares to control run variance $\left(\operatorname{RSS} /\left(N \cdot \operatorname{Var}_{\text {cntrl }}\right)\right)$ are $0.93,1.01,1.29$, and 1.13 , respectively. Although ratios of explained variation for cloudiness, precipitation, and logarithmic precipitation appear to be very small, the comparison of residual variance to the control run variance reveals that most of the unexplained variation can be attributed to the high interannual variability of these variables. This is a clear indication that the derived patterns have a strong significance and can be used in a scenario-building framework such as the one applied here. Even the relatively high value of $\left(\mathrm{RSS} /\left(N \cdot \operatorname{Var}_{\text {cntrl }}\right)\right)$ for increasing precipitation (1.29) is not critical if one considers that increases of mean precipitation are usually accompanied by increases in variability. Because a transformation to logarithmic values diminishes this effect, the ratio of residual variance to control run variance is very close 
Table 2. Overview of individual AOGCMs' ratios of explained sum squares (ESS) to total sum of squares (TSS) and ratios of residual sum of squares (RSS) to scaled control run variance $\left(N \cdot \operatorname{Var}_{\text {cntrl }}\right)$ for temperature, cloudiness, increasing precipitation, and decreasing logarithmic precipitation. In all cases, only significant linear regression models are included. The scaling of control run variance is necessary to make it comparable to RSS, which is calculated for $N$ values.

\begin{tabular}{|c|c|c|c|c|c|c|c|c|}
\hline & \multicolumn{2}{|c|}{ Temperature } & \multicolumn{2}{|c|}{ Cloudiness } & \multicolumn{2}{|c|}{$\begin{array}{l}\text { Increasing } \\
\text { Precipitation }\end{array}$} & \multicolumn{2}{|c|}{$\begin{array}{l}\text { Decreasing Log. } \\
\text { Precipitation }\end{array}$} \\
\hline & ESS/TSS & $\mathrm{RSS} /\left(N \cdot \operatorname{Var}_{\text {cntrl }}\right)$ & ESS/TSS & $\mathrm{RSS} /\left(N \cdot \operatorname{Var}_{\mathrm{cntrl}}\right)$ & ESS/TSS & $\mathrm{RSS} /\left(N \cdot \operatorname{Var}_{\mathrm{cntrl}}\right)$ & ESS/TSS & $\mathrm{RSS} /\left(N \cdot \operatorname{Var}_{\mathrm{cntrl}}\right)$ \\
\hline CCCMA-CGCM3.1 & 0.86 & 0.88 & 0.28 & 0.92 & 0.19 & 1.31 & 0.18 & 1.09 \\
\hline CNRM-CM3 & 0.81 & 0.87 & 0.21 & 0.96 & 0.15 & 1.28 & 0.16 & 1.14 \\
\hline CSIRO-MK3.0 & 0.72 & 0.98 & 0.20 & 1.34 & 0.10 & 1.23 & 0.12 & 1.12 \\
\hline GFDL-CM2.0 & 0.69 & 0.99 & 0.18 & 1.04 & 0.12 & 1.37 & 0.13 & 1.18 \\
\hline GFDL-CM2.1 & 0.70 & 0.98 & 0.18 & 1.01 & 0.10 & 1.30 & 0.16 & 1.15 \\
\hline GISS-EH & 0.66 & 0.89 & 0.18 & 1.01 & 0.24 & 1.20 & 0.21 & 1.03 \\
\hline GISS-ER & 0.74 & 0.96 & 0.16 & 0.97 & 0.25 & 1.26 & 0.16 & 1.03 \\
\hline IAP-FGOALS-g1.0 & 0.70 & 0.82 & 0.11 & 0.93 & 0.15 & 1.09 & 0.09 & 0.96 \\
\hline INM-CM3.0 & 0.72 & 0.89 & 0.15 & 0.96 & 0.15 & 1.20 & 0.13 & 1.09 \\
\hline IPSL-CM4 & 0.83 & 0.95 & 0.32 & 1.00 & 0.27 & 1.53 & 0.18 & 1.20 \\
\hline MIROC3.2(hires) & 0.86 & 0.98 & 0.28 & 1.00 & 0.14 & 1.40 & 0.13 & 1.17 \\
\hline MIROC3.2(medres) & 0.86 & 0.97 & 0.28 & 1.01 & 0.17 & 1.37 & 0.13 & 1.15 \\
\hline MIUB-ECHO-G & 0.87 & 0.89 & 0.21 & 0.97 & 0.24 & 1.38 & 0.16 & 1.22 \\
\hline MPI-ECHAM5 & 0.79 & 1.00 & 0.14 & 1.05 & 0.11 & 1.34 & 0.11 & 1.21 \\
\hline MRI-CGCM2.3.2a & 0.82 & 1.01 & 0.23 & 1.01 & 0.14 & 1.25 & 0.13 & 1.15 \\
\hline NCAR-CCSM3 & 0.82 & 0.88 & 0.19 & 1.00 & 0.20 & 1.21 & 0.12 & 1.13 \\
\hline NCAR-PCM1 & 0.77 & 0.82 & 0.11 & 1.01 & 0.14 & 1.15 & 0.08 & 1.10 \\
\hline UKMO-HadCM3 & 0.79 & 0.98 & 0.25 & 1.01 & 0.15 & 1.33 & 0.22 & 1.22 \\
\hline UKMO-HadGEM1 & 0.81 & 0.95 & 0.19 & 1.00 & 0.13 & 1.34 & 0.19 & 1.19 \\
\hline all & 0.81 & 0.92 & 0.21 & 1.00 & 0.17 & 1.30 & 0.14 & 1.14 \\
\hline $\min$ & 0.66 & 0.82 & 0.11 & 0.92 & 0.10 & 1.09 & 0.08 & 0.96 \\
\hline $\max$ & 0.87 & 1.01 & 0.32 & 1.34 & 0.27 & 1.53 & 0.22 & 1.22 \\
\hline median & 0.79 & 0.95 & 0.19 & 1.00 & 0.15 & 1.30 & 0.13 & 1.15 \\
\hline mean & 0.78 & 0.93 & 0.20 & 1.01 & 0.16 & 1.29 & 0.15 & 1.13 \\
\hline
\end{tabular}

to unity (0.98) if it is calculated for increasing logarithmic precipitation. It should be mentioned, however, that precipitation change in the AOGCM simulations is also influenced by factors such as atmospheric aerosol loading, as these effects are not captured by the extracted patterns and therefore contribute to higher $\left(\mathrm{RSS} /\left(N \cdot \operatorname{Var}_{\mathrm{cntrl}}\right)\right)$ ratios. The ratio of residual variance to control run variance smaller than unity for temperature means that the residual variation is generally slightly smaller than expected from the interannual variability estimated from the control run. This is an indicator for the strong relationship between local temperature anomalies and $T_{\text {glob }}$ anomalies captured by the derived patterns. When using these patterns to predict local temperature anomalies in conjunction with actual $\Delta T_{\text {glob }}(y)$, the part of interannual variability that can be explained by interannual variability of $\Delta T_{\text {glob }}(y)$ is included which reduces the residual error. In contrast, the estimation of control run variance is based on a constant mean climatology and therefore includes the part of variability that is correlated to the variability in $\Delta T_{\mathrm{glob}}(y)$.

\subsection{Applied local anomalies for 1 degree of global warming}

The dataset for systematic climate impact assessment presented here is a combination of extracted patterns and the reference time series of temperature, precipitation, and cloudiness. While properties of the scaling patterns were discussed in the preceding section, this section explores the actual anomalies by which the scenario time series are shifted. For each variable the scaling patterns that represent the anomalies for a 1-degree increase in $T_{\text {glob }}$ are applied to the reference time series according to the methodology described in Sect. 2.3). Thereby, the absolute change $V^{*}(x, m) \cdot 1 \mathrm{~K}$ is altered, depending on the application method and the degree of disagreement between observed and simulated present-day climate. From the obtained time series multi-model means of the actual applied annual mean change are calculated:

$\overline{\Delta \bar{V}}_{\mathrm{appl}, 1 \mathrm{~K}}(x)=\frac{1}{19 \cdot 12} \sum_{i=1}^{19} \sum_{m=1}^{12}\left[\bar{V}_{\mathrm{scen}, 1 \mathrm{~K}}(x, m, i)-\bar{V}_{\mathrm{ref}}(x, m)\right]$,

where $\bar{V}_{\text {scen, } 1 \mathrm{~K}}(x, m, i)$ is the long-term climatological mean of the scenario time series for a $T_{\text {glob }}$ increase by $1 \mathrm{~K}$ in location $x$, month $m$, and AOGCM $i$.

The alteration of anomalies by the application procedure is an important aspect of the methodology described in this paper. It is, however, a very general problem how to interpret and apply AOGCM-derived changes in climatological means when these means are biased. If the observed climatology is underestimated the simulated change may underestimate the actual change and vice versa, providing that changes derived from a biased representation of reality are a meaningful source of actual change at all. All assessments that are based on anomalies obtained from AOGCM simulations are confronted with this problem and have to deal with the question 
whether to use the unchanged absolute anomalies or adjust them according to the biases in the AOGCM's presentation of actual conditions. In cases where anomalies are combined with observations, an adjustment is often inevitable, as a direct use of anomalies can cause an exceedance of valid ranges for some variables (e.g. most variables have a positivity constraint). In these cases a relative application of anomalies provides a convenient and plausible way of accounting for the different base levels in simulations and observations. There are, however, no objective criteria on whether and how to perform this adjustment. Hence, any solution represents a choice that cannot be validated in a meaningful way. Our methodology is no exception from that. It is grounded on common practice found in the impact literature, aiming to fulfil the particular requirements of the pattern-scaling approach, while minimising alterations of the original signal. In place of a validation, we here complement the presentation of applied anomalies in the end product by a presentation of the alteration of the original anomalies. Multi-model means of the alteration of the original anomalies $V^{*}(x, m) \cdot 1 \mathrm{~K}$ in $\bar{V}_{\text {scen,1 } \mathrm{K}}(x, m, i)$ are calculated as

$$
\begin{aligned}
\bar{\Delta}_{\text {alt }, 1 \mathrm{~K}}(x) & =\frac{1}{19 \cdot 12} \sum_{i=1}^{19} \sum_{m=1}^{12}\left[\mid \bar{V}_{\text {scen }, 1 \mathrm{~K}}(x, m, i)\right. \\
& \left.-\bar{V}_{\mathrm{ref}}(x, m)|-| V^{*}(x, m, i) \cdot 1 \mathrm{~K} \mid\right] .
\end{aligned}
$$

The omission of the sign of change by the modulo function in Eq. (17) ensures that augmentations always have a positive sign and attenuations always have an negative sign, regardless of the sign of change.

For temperature, the actual applied anomalies for a 1degree increase in $T_{\text {glob }}$ (Fig. 3) are identical to the scaling pattern, as temperature anomalies are applied as absolute changes (Eq. 7). The spatial distribution of mean annual temperature changes across all AOGCMs exhibits the same overall behaviour as presented and discussed for the CMIP3 ensemble in Solomon et al. (2007). For the considered land area there are no incidents of decreasing local temperature with increasing $T_{\text {glob. }}$. Below average warming (green colours) is only found in the vicinity of oceans, which is the result of the thermal inertia of the oceans. Overall, warming on the land surface is above average with a distinct pattern of polar amplification (stronger warming towards higher latitudes). Behind the multi-model annual mean change there is substantial variation in regional temperature change both among different AOGCMs and during the course of the year (see Supplement). Disparity among AOGCMs is lower than the projected mean change - i.e. there is some disagreement in the magnitude but not in the direction of change. Seasonality of change is particularly strong in the high northern latitudes and broadly follows the pattern of polar amplification. Hence, the strong average increase projected for these areas does not occur uniformly over the year.

Actual applied anomalies for cloudiness are a mix of cloud cover increases and decreases (Fig. 4). Strong decreases
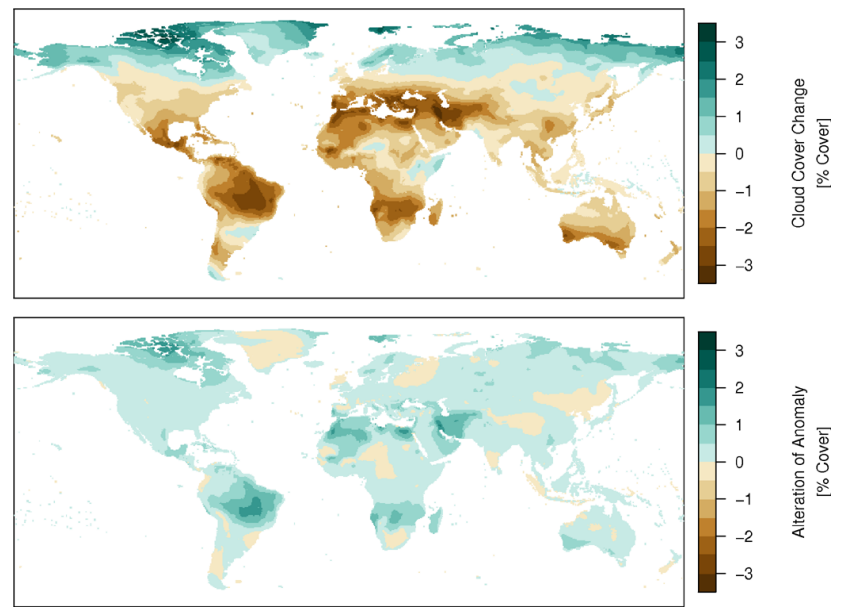

Fig. 4. Upper panel: multi-model mean of the actual applied annual mean change in cloudiness in $\%$ cloud cover per $1 \mathrm{~K}$ of $\Delta T_{\text {glob }}$ (Eq. 16). Lower panel: multi-model mean of the alteration of the original anomaly in $\%$ cloud cover for $1 \mathrm{~K}$ of $\Delta T_{\text {glob }}$ (Eq. 17); positive values indicate an augmentation and negative values indicate an attenuation, regardless of the direction of change.

are found in the Mediterranean, the Middle East, southern Africa, southern Australia, Central America, and the Amazon region. Increases are constrained to the higher northern latitudes and the Horn of Africa. In some areas, such as the northernmost latitudes, the Amazon, and some parts of Africa, variation of projected annual cloud cover change among AOGCMs is high with inter-model standard deviation exceeding the mean change (see Supplement). Significant seasonality in the multi-model mean is limited to a few regions such as the Amazon, Central Asia and northeastern Canada only (see Supplement). Regions with pronounced seasonality do not always coincide with regions of strong mean change, which indicates a mix of increases and decreases throughout the year that cancel out each other in the annual mean.

Alteration of the absolute signal, averaged over all months and AOGCMs, by the application method described in Sect. 2.3.2 is depicted in the lower panel of Fig. 4. In most cases the application method augments the original signal, which means that decreases of cloudiness tend to be associated by underestimation and increases by overestimation of present-day cloud cover. However, in most cases the average alteration of the original signal is less than $\pm 0.5 \%$. Significant alteration of the signal only occurs in northern Canada, the Amazon, the Middle East, and some parts of Africa - all of these regions being characterised by strong mean changes (Fig. 4, upper panel).

The multi-model mean of annual precipitation change is shown in Fig. 5 (upper panel). As for temperature and cloudiness, precipitation changes are consistent with results presented in Solomon et al. (2007). Significant decreases prevail in the Mediterranean, the Middle East, South 

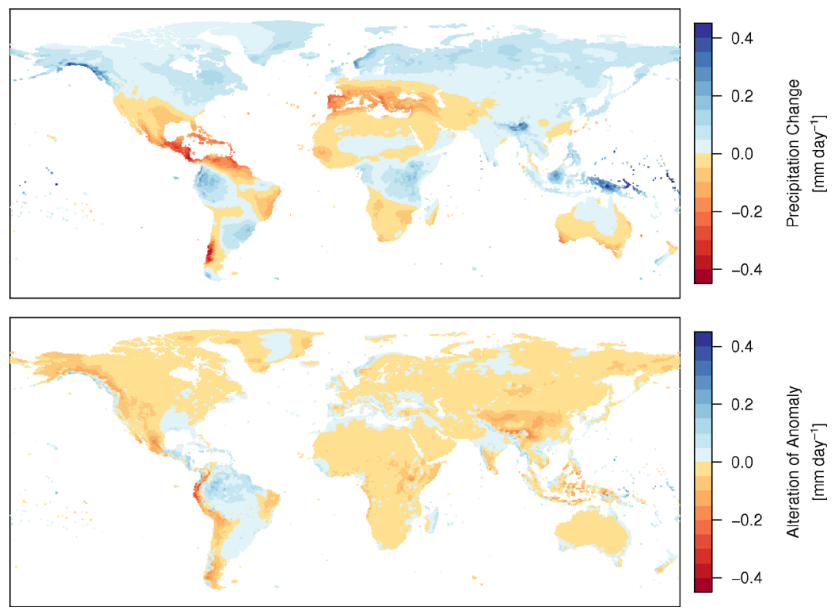

Fig. 5. Upper panel: multi-model mean of the actual applied annual mean change in precipitation rate in $\mathrm{mm} \mathrm{d}^{-1}$ per $1 \mathrm{~K}$ of $\Delta T_{\text {glob }}$ (Eq. 16). Lower panel: multi-model mean of the alteration of the original anomaly in $\mathrm{mm} \mathrm{d}^{-1}$ for $1 \mathrm{~K}$ of $\Delta T_{\text {glob }}$ (Eq. 17); positive values indicate an augmentation and negative values indicate an attenuation, regardless of the direction of change.

Africa, southern Australia, Central America and Patagonia; increases are projected for the Boreal zone, South and Southeast Asia, East Africa, and parts of South America. For some regions such as the Amazon, Sub-Saharan Africa, and Southeast Asia inter-model standard deviation is high (see Supplement), indicating considerable disagreement in the magnitude and in some cases even sign of mean annual precipitation change for the different AOGCMs. Seasonality of change is less pronounced but seems to occur in regions where the inter-model spread is high - i.e. the wet tropics but also in temperate North America and Europe (see Supplement).

Although large biases in the AOGCMs impair the applicability of derived anomalies the alteration of the scaled anomalies by the application method is well controlled and rarely exceeds $\pm 0.05 \mathrm{~mm} \mathrm{~d}^{-1}$. Significant alterations primarily occur in mountainous regions (Andes, Rocky Mountains, Himalayas) where the AOGCMs' coarse spatial resolution impedes the correct representation of sub-grid orographic effects. In average, our application method attenuates rather than augments the original anomaly, which indicates that AOGCMs tend to overestimate observed precipitation rates. It is not the progressive reduction of the relative anomaly by the $\lambda$ exponent with increasing underestimation in the AOGCM (Eq. 9) that causes the overall attenuation. The reduction of the relative anomaly applies to both increases and decreases and merely compensates for the asymmetry in the relative application of anomalies derived from differently biased AOGCM baselines. While the attenuation in case of overestimation can never exceed the original anomaly when applied as relative change, the augmentation in case of underestimation in the AOGCM can become many times bigger
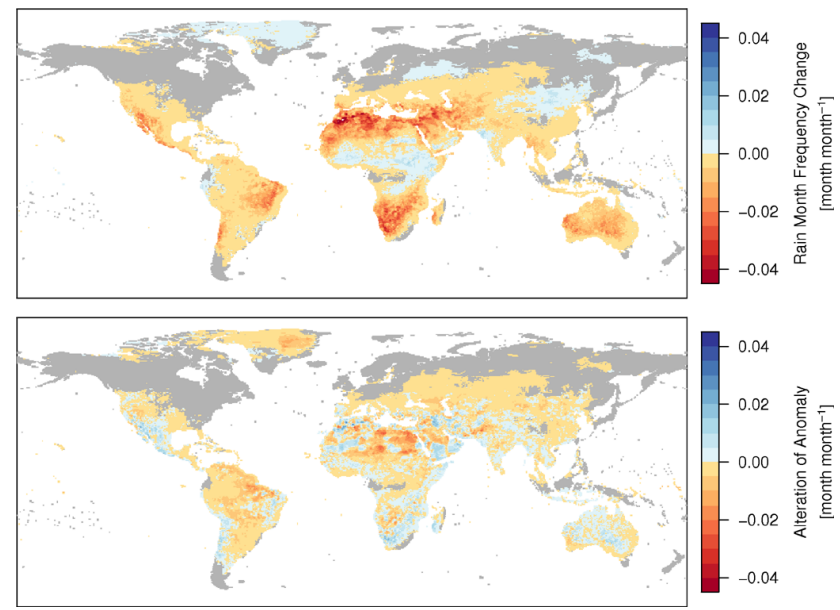

Fig. 6. Upper panel: multi-model mean of the actual applied annual mean change in rain month frequency in month/month for a $\Delta T_{\text {glob }}$ of $1 \mathrm{~K}$ (Eq. 16). Lower panel: multi-model mean of the alteration of the original anomaly in month/month for $1 \mathrm{~K}$ of $\Delta T_{\text {glob }}$ (Eq. 17); positive values indicate an augmentation and negative values indicate an attenuation, regardless of the direction of change.

than the original anomaly. With our approach, in contrast, the original anomaly is also augmented with increasing underestimation in the AOGCM, but reaches a maximum augmentation by a factor of about two for a five-fold underestimation and then declines towards unity for a completely rain-free AOGCM baseline.

Changes in rain month frequency are rarely analysed and here their explicit consideration in a pattern-scaling framework is unique. The rain month frequency changes, averaged over all AOGCMs and months, shown in the upper panel of Fig. 6, exhibit both increases and decreases although decreases prevail. As already discussed in Sect. 3.1, changes occur predominately in areas that are already today characterised by intermittent rainfall occurrence while regions such as North America, northern Europe, and Siberia remain unaffected. Regions of strong rain month frequency decrease broadly agree with key regions of decreases in average rainfall, but some noteworthy differences exist. Almost entire South America and Australia are, on average, affected by rain month frequency decrease while the picture for change in rainfall amount is much more mixed. In the Mediterranean, southern Europe is much less affected by rainfall amounts, while the opposite can be stated for North Africa. In southern Africa decreases in rain month frequency stretch much further up north along the east coast.

Variation of rain month frequency change among AOGCMs is pronounced but generally follows the pattern of strong decreases (see Supplement). Thus, different models disagree primarily in the magnitude rather than in the direction of change. Seasonality of change is in the same magnitude as the inter-model variation and also exhibits a similar pattern (see Supplement). Hence, decreases in rain month 
frequency in some months can be very high, while little change occurs in others.

Anomalies of rain month frequency are significantly altered by the application method (see Fig. 6, lower panel). Although logit-transformed frequency anomalies are applied as absolute changes (see Sect. 2.3.4), the different reference levels in the AOGCM and the observations result in very different actual frequency anomalies when transformed back. Equation (11) implies a sigmoid shape for the relationship between rain month frequency and $\Delta T_{\text {glob }}$, which means that a given $\beta^{*} \cdot \Delta T_{\text {glob }}(y)$ produces the strongest change in rain month frequency when applied to a rain month frequency of 0.5 ; with reference values closer to 0 and 1 the effect progressively decreases. Consequently, augmentations of the signal occur when frequencies in the AOGCM are close to 0 or 1 and projected changes are applied to observed rain month frequencies closer to 0.5. Attenuations occur in cases where changes are estimated from intermediate rain month frequency levels in the AOGCM and applied to reference frequencies closer to 0 or 1.

In summary, the multi-model mean of applied annual change for the different variables presented here are - where applicable - consistent with the results presented in Solomon et al. (2007). Although the application method can significantly alter the absolute anomaly for some variables, these alterations are not arbitrary but a consequence of the biases in AOGCMs. We believe that the application methods chosen for the different climate variables are well justified and fulfil the aim of providing the necessary adjustment while minimizing unnecessary alterations.

\section{Conclusions}

Here we present a newly composed dataset of climate change scenarios for systematic assessments of climate change impacts as a function of $T_{\text {glob }}$ increase. The dataset combines observations, information extracted from AOGCM simulations, and results from a reduced complexity climate model into physically plausible climate change scenarios for a wide range of global mean temperature increases. The scenarios are designed to reach global mean temperature increases above pre-industrial levels between 1.5 and 5 degrees (in 0.5 degree steps) around the year 2100 . The scaling patterns extracted for 19 AOGCMs from the CMIP3 data base for temperature, cloudiness, and precipitation represent the key component for linking local climate change to changes in $T_{\text {glob }}$. We discuss the properties of these patterns and demonstrate that they preserve the original AOGCM climate change properties with sufficient accuracy. The methodology for combining the local climate anomalies (derived from the scaling patterns and $\Delta T_{\text {glob }}$ trajectories) with observations is extensively discussed as it has the potential to alter the derived raw anomalies. We show that alterations of climate anomalies by the application method are a necessary adjustment of anomalies obtained from biased AOGCM baselines. The additional material used for creating the dataset - global datasets on observed historical climate and the reduced complexity climate model MAGICC6 - are not further discussed in this paper. They are well documented in other literature.

\section{Supplementary material related to this article is

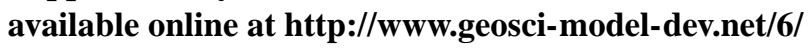 1689/2013/gmd-6-1689-2013-supplement.pdf.}

Acknowledgements. This work was supported by ILRI and the CGIAR research program on Climate Change, Agriculture and Food Security (CCAFS). Further support was given by GLUES (Global Assessment of Land Use Dynamics, Greenhouse Gas Emissions and Ecosystem Services), a scientific coordination and synthesis project of the German Federal Ministry of Education and Research's (BMBF's) "Sustainable Land Management" programme (Code 01LL0901A), and the WATCH (FP6, grant no. 036946), CLIMAFRICA (FP7, grant no. 244240) and ERMITAGE (FP7, grant no. 265170) projects funded by the European Commission.

We acknowledge the modelling groups, the Programme for Climate Model Diagnosis and Intercomparison (PCMDI) and the WCRP's Working Group on Coupled Modelling (WGCM) for their roles in making available the WCRP CMIP3 multi-model dataset. Support of this dataset is provided by the Office of Science, US Department of Energy.

Edited by: M. Kawamiya

\section{References}

Arnell, N. W., van Vuuren, D. P., and Isaac, M.: The implications of climate policy for the impacts of climate change on global water resources, Global Environ. Chang., 21, 592-603, 2011.

Carter, T., Parry, M. L., Harasawa, H., and Nishioka, S.: IPCC Technical Guidelines for Assessing Impacts of Climate Change, Tech. Rep. IPCC Special Report 0904813118, Intergovernmental Panel on Climate Change, WMO and UNEP, 1994.

Füssel, H.-M.: Impacts analysis for inverse integrated assessments of climate change, Ph.D. thesis, Potsdam, Germany, 2003.

Gerber, S., Joos, F., and Prentice, I. C.: Sensitivity of a dynamic global vegetation model to climate and atmospheric $\mathrm{CO}_{2}$, Glob. Change Biol., 10, 1223-1239, doi:10.1111/j.15298817.2003.00807.x, 2004.

Gerten, D., Heinke, J., Hoff, H., Biemans, H., Fader, M., and Waha, K.: Global Water Availability and Requirements for Future Food Production, J. Hydrometeorol., 12, 885-899, 2011.

Gosling, S. N., Bretherton, D., Haines, K., and Arnell, N. W.: Global hydrology modelling and uncertainty: running multiple ensembles with a campus grid, Philos. T. R. Soc. A, 368, 4005-4021, 2010.

Harris, I., Jones, P. D., Osborn, T. J., and Lister, D. H.: Updated high-resolution grids of monthly climatic observations 
- the CRU TS3.10 Dataset, Int. J. Climatol., online first, doi:10.1002/joc.3711, 2013.

Hawkins, E. and Sutton, R.: The potential to narrow uncertainty in regional climate predictions, B. Am. Meteorol. Soc., 90, 10951107, 2009.

Hawkins, E. and Sutton, R.: The potential to narrow uncertainty in projections of regional precipitation change, Clim. Dynam., 37, 407-418, 2011.

Heyder, U., Schaphoff, S., Gerten, D., and Lucht, W.: Risk of severe climate change impact on the terrestrial biosphere, Environ. Res. Lett., 6, 034-036, 2011.

Hulme, M., Jiang, T., and Wigley, T.: SCENGEN: A Climate Change SCENario GENerator: Software User Manual, Version 1.0, Tech. rep., Climate Change Research Unit, School of Environmental Sciences, University of East Anglia, 1995.

Huntingford, C. and Cox, P. M.: an analogue model to derive additional climate change scenarios from existing GCM simulations, Clim. Dynam., 16, 575-586, 2000.

Lambert, F. H. and Chiang, J. C. H.: Control of land-ocean temperature contrast by ocean heat uptake, Geophys. Res. Lett., 34, L13704, doi:10.1029/2007GL029755, 2007.

Mann, M. E.: Defining dangerous anthropogenic interference, P. Natl. Acad. Sci., 106, 4065-4066, 2009.

Meehl, G. A., Covey, C., Delworth, T., Latif, M., McAvaney, B., Mitchell, J. F. B., Stouffer, R. J., and Taylor, K. E.: The WCRP CMIP3 Multimodel Dataset: A new era in climate change research, B. Am. Meteorol. Soc., 88, 1383-1394, doi:10.1175/BAMS-88-9-1383, 2007.

Meinshausen, M., Hare, B., Wigley, T. M. L., van Vuuren, D. P., den Elzen, M. G. J., and Swart, R.: Multi-gas emission pathways to meet climate targets, Climatic Change, 75, 151-194, 2006.

Meinshausen, M., Meinshausen, N., Hare, W., Raper, S. C. B., Frieler, K., Knutti, R., Frame, D. J., and Allen, M. R.: Greenhouse-gas emission targets for limiting global warming to $2{ }^{\circ} \mathrm{C}$, Nature, 458, 1158-1162, doi:10.1038/nature08017, 2009.

Meinshausen, M., Raper, S. C. B., and Wigley, T. M. L.: Emulating coupled atmosphere-ocean and carbon cycle models with a simpler model, MAGICC6 - Part 1: Model description and calibration, Atmos. Chem. Phys., 11, 1417-1456, doi:10.5194/acp11-1417-2011, 2011a.

Meinshausen, M., Smith, S., Calvin, K., Daniel, J., Kainuma, M., Lamarque, J.-F., Matsumoto, K., Montzka, S., Raper, S., Riahi, K., Thomson, A., Velders, G., and van Vuuren, D.: The RCP greenhouse gas concentrations and their extensions from 1765 to 2300 , Climatic Change, 109, 213-241, doi:10.1007/s10584011-0156-z, 2011b.

Mitchell, T. D.: Pattern Scaling: An Examination of the Accuracy of the Technique for Describing Future Climates, Climatic Change, 60, 217-242, 2003.

Müller, C., Cramer, W., Hare, W. L., and Lotze-Campen, H.: Climate change risks for African agriculture, P. Natl. Acad. Sci. USA, 108, 4313-4315, 2011.

Murray, S. J., Foster, P. N., and Prentice, I. C.: Future global water resources with respect to climate change and water withdrawals as estimated by a dynamic global vegetation model, J. Hydrol., 448-449, 14-29, 2012.
New, M., Hulme, M., and Jones, P.: Representing twentieth-century space-time climate variability. Part II: Development of 19011996 monthly grids of terrestrial surface climate, J. Climate, 13, 2217-2238, 2000.

New, M., Liverman, D., Schroder, H., and Anderson, K.: Four degrees and beyond: the potential for a global temperature increase of four degrees and its implications, Philos. T. R. Soc. A, 369, 6-19, 2011.

Parry, M., Canziani, O., Palutikof, J., Linden, P. v. d., and Hanson, C.: Climate Change 2007: Impacts, Adaptation and Vulnerability. Contribution of Working Group II to the Fourth Assessment Report of the Intergovernmental Panel on Climate Change, Cambridge University Press, Cambridge, UK, 2007.

Ramanathan, V. and Feng, Y.: On avoiding dangerous anthropogenic interference with the climate system: Formidable challenges ahead, P. Natl. Acad. Sci., 105, 14245-14250, doi:10.1073/pnas.0803838105, 2008.

R Development Core Team: R: A Language and Environment for Statistical Computing, R Foundation for Statistical Computing, Vienna, Austria, 2011.

Rudolf, B., Becker, A., Schneider, U., Meyer-Christoffer, A., and Ziese, M.: GPCC Status Report December 2010 (On the most recent gridded global data set issued in fall 2010 by the Global Precipitation Climatology Centre (GPCC)), Tech. rep., DWD/GPCC, 2010.

Scholze, M., Knorr, W., Arnell, N. W., and Prentice, I. C.: A climate-change risk analysis for world ecosystems, P. Natl. Acad. Sci., 103, 13116-13120, 2006.

Sitch, S., Huntingford, C., Gedney, N., Levy, P. E., Lomas, M., Piao, S. L., Betts, R., Ciais, P., Cox, P., Friedlingstein, P., Jones, C. D., Prentice, I. C., and Woodward, F. I.: Evaluation of the terrestrial carbon cycle, future plant geography and climate-carbon cycle feedbacks using five Dynamic Global Vegetation Models (DGVMs), Glob. Change Biol., 14, 2015-2039, 2008.

Smith, J. B., Schneider, S. H., Oppenheimer, M., Yohe, G. W., Hare, W., Mastrandrea, M. D., Patwardhan, A., Burton, I., CorfeeMorlot, J., Magadza, C. H. D., Füssel, H.-M., Pittock, A. B., Rahman, A., Suarez, A., and van Ypersele, J.-P.: Assessing dangerous climate change through an update of the Intergovernmental Panel on Climate Change (IPCC) "reasons for concern", P. Natl. Acad. Sci. USA, 106, 4133-4137, 2009.

Solomon, S., Qin, D., Manning, M., Chen, Z., Marquis, M., Averyt, K. B., Tignor, M., and Miller, H. L.: Climate Change 2007: The Physical Science Basis. Contribution of Working Group I to the Fourth Assessment Report of the Intergovernmental Panel on Climate Change, Cambridge University Press, Cambridge, UK, 2007.

Solomon, S., Plattner, G.-K., Knutti, R., and Friedlingstein, P.: Irreversible climate change due to carbon dioxide emissions, P. Natl. Acad. Sci., 106, 1704-1709, 2009.

Watterson, I. G.: Calculation of probability density functions for temperature and precipitation change under global warming, J. Geophys. Res-Atmos., 113, D12106, doi:10.1029/2007JD009254, 2008. 